\title{
Caesarean Section of Multifetal Pregnancy
}

\author{
Shinji Tanigaki ${ }^{1}$ Satoshi Takemori ${ }^{1}$ Makoto Osaka ${ }^{1}$ Momoe Watanabe ${ }^{1}$ Aya Kitamura ${ }^{1}$ \\ Sayaka Ueyama ${ }^{1}$ Kei Tanaka ${ }^{1}$ Miho Matsushima ${ }^{1}$ Youichi Kobayashi ${ }^{1}$ \\ ${ }^{1}$ Department of Obstetrics and Gynecology, Kyorin University School \\ of Medicine, Mitaka City, Tokyo, Japan \\ Address for correspondence Shinji Tanigaki, MD, PhD, Department of \\ Obstetrics and Gynecology, Kyorin University School of Medicine, \\ 6-20-2 Shinkawa, Mitaka City, Tokyo 181-8611, Japan \\ Surg J 2020;6(suppl S2):S92-S97. \\ (e-mail: tanigaki@ks.kyorin-u.ac.jp).
}

Abstract
Keywords
- multifetal pregnancy
- twin pregnancy
- caesarean section
- postpartum
hemorrhage

Planned caesarean delivery (CD) did not significantly decrease or increase the risk of fetal or neonatal death or serious neonatal morbidity in twin pregnancy between $32^{\text {0/7 }}$ and $38^{6 / 7}$ weeks of gestation, with the first twin in the vertex presentation. As prevalence rises for the second twin, emergency $C D$ is necessary for delivery of the second twin after vaginal delivery of the first twin. Waiting after 38 weeks' gestation essentially requires close fetal and maternal surveillance to identify if those pregnancies may benefit to extend a gestational period. It is important to construct a system in which an emergency CD can be performed anytime. The caesarean section does not change in even multifetal pregnancy. Each step after laparotomy has few tips: (1) because the uterus strongly leans to the right, image the uterine rotation. To avoid thick vessels on the uterine lateral wall, perform long U-shaped incision using a scissor. 2) Ensure not to rupture the membrane of the second twin before delivery of the first twin. (3) Check the presentation of the second twin before rupture of that fetus's membrane. The second twin tends to change the presentation. If the upper uterine segment will clamp down and entrap the second twin, a vertical uterine incision is performed without hesitation. Women with multifetal pregnancy are at increased risk of postpartum hemorrhage (PPH). Mainly PPH is caused by uterine atony. Oxytocin should be prepared before starting the $\mathrm{CD}$. All bleeding may not be recognized in the operation field. Do not lose the timing of blood transfusion.

\section{Selection of Surgical Technique}

Indications of Caesarean Delivery for Twin Pregnancy Twin pregnancy is associated with increased perinatal mortality than singleton pregnancy because of complications during birth. Higher rates of adverse perinatal outcome have been reported for the twin pregnancy at full or near term if born by vaginal (VD) versus caesarean delivery (CS). ${ }^{1}$ But there was no clear evidence of differences between planned VD and CS for maternal death or serious morbidity (risk ratio $[R R]=0.86$, 95\% confidence interval $[\mathrm{CI}]: 0.67-1.11$ ), and there was no significant difference for perinatal or neonatal death or serious neonatal morbidity between planned VD and CS $(R R=1.15$, 95\% CI: 0.80-1.67). ${ }^{2}$ However, monochorionic, monoamniotic twins are avoided VD because of anxiety for tangled umbilical cords. Currently, if the operator is not experienced in vaginal breech delivery, $\mathrm{CD}$ is performed even for singleton pregnancy. Additionally, breech-vertex presentation is capable of occurring interlocking. It is generally performed in planned $C D$ in which the first twin is in nonvertex presentation. Although there is no evidence that VD of fetus in vertex presentation weighing less than $1,500 \mathrm{~g}$ is associated with any increased risk of perinatal mortality, planned $\mathrm{CD}$ is recommended for lowbirth weight twin and preterm delivery prior to 34 weeks' gestation. If the first twin is in vertex presentation and fetuses weighing at least $1,500 \mathrm{~g}$ or reaching at least 32 weeks' gestation, a low 5-minute Apgar's score occurred less frequently by planned $\mathrm{CS}^{3}$ In twin pregnancy, between $32^{0 / 7}$ and $38^{6 / 7}$ weeks of gestation, with the first twin in the vertex presentation, planned CD did not significantly decrease or increase the
DOI https://doi.org/ 10.1055/s-0040-1712924. ISSN 2378-5128.
Copyright @ 2020 by Thieme Medical Publishers, Inc., 333 Seventh Avenue, New York, NY 10001, USA. Tel: +1(212) 760-0888.
License terms

(๑) (1) $\Theta$ 
Table 1 Indications of cesarean delivery for twin pregnancy

\begin{tabular}{|l|l|}
\hline Chorionicity & Monochorionic monoamniotic twin \\
\hline $\begin{array}{l}\text { Gestational } \\
\text { age (wk) }\end{array}$ & $<32$ \\
\hline Presentation & $\begin{array}{l}\text { The first twin in the nonvertex } \\
\text { presentation }\end{array}$ \\
\hline $\begin{array}{l}\text { Estimated fetal } \\
\text { weight }(\mathrm{g})\end{array}$ & $<1,500$ \\
\cline { 2 - 2 } $\begin{array}{l}\text { Present of cesarean delivery indications for singleton } \\
\text { pregnancy }\end{array}$ \\
\hline $\begin{array}{l}\text { Shortage of human resources, materials, and informed } \\
\text { consents }\end{array}$ \\
\hline
\end{tabular}

(Reproduced with permission from Tanigaki S, Iwashita M. Multifetal pregnancy. In: Hiramatsu Y, Konishi I, Sakuragi N, Takeda S, eds. Mastering the Essential Surgical Procedures OGS NOW, No.3. Cesarean Section (Japanese). Tokyo: Medical View; 2010: 56-63. Copyright ( Medical View).

risk of fetal or neonatal death or serious neonatal morbidity, as compared with planned VD. ${ }^{4}$ Indications of $\mathrm{CD}$ is given in - Table 1.

\section{Timing of Planned CD}

Minakami et al reported that the incidence of stillbirth and early neonatal death gradually declined until 37 to 38 weeks' gestation and then increased. So, limiting the estimated date of delivery from 37 to 38 weeks may be appropriate in multifetal pregnancies. ${ }^{5}$ Luke reported that in the United States, the lowest fetal death rates in twin pregnancies occurred at 36 to 37 week's gestation, too. ${ }^{6}$ On the other hand, there were significantly many neonates with respiratory disorders in which those have undergone CD labor before 38 weeks' gestation than those after 38 weeks' gestation. ${ }^{7}$ In this article, it is noted that avoiding $C D$ until the onset of labor or until after 38 weeks' gestation should be considered. As gestational weeks progress, the risk of preterm delivery is sharply increases. The odds ratio for delivery within 1 week in multifetal pregnancies compared with singleton pregnancies was 5.9 (95\% CI: 5.4-6.5) at 22 weeks'

The procedures of caesarean section in twin pregnancy

Laparotomy
Delivery of the first twin
Prevention of PPH
Wound closure

Fig. 1 Surgical steps. The procedures of caesarean section in twin pregnancy. PPH, postpartum hemorrhage. gestation and 13.7 (95\% CI: 13.1-14.2) at 33 weeks' gestation. ${ }^{8}$ Thus progressing weeks lead to the increasing possibility of emergency $\mathrm{CD}$. In addition, women pregnant with multifetal pregnancy have significantly greater and more severe pregnancy-related complications, such as hypertensive disorder of pregnancy, HELLP syndrome (hemolysis, elevated liver enzymes, low platelet count), cardiomyopathy, thrombosis, and others. Therefore in multifetal pregnancy, waiting after 38 weeks' gestation essentially requires close fetal and maternal surveillance to identify if those pregnancies may benefit to extend a gestational period. It is important to construct the system that an emergency CD can perform anytime.

\section{Surgical Steps}

The procedure of CD in twin pregnancy shows in - Fig. 1 . The $C D$ does not change in even multifetal pregnancy. Each step after laparotomy has few tips.

\section{In-Depth Explanation}

\section{Myometrium Incision-Imaging of Uterine Rotation and Long U-Shaped Incision}

In multifetal pregnancy, the uterus strongly leans to the right to put the uterus which enlarged excessively in a pelvis (dextrorotation). The authors incise myometrium after a pause, and then the round ligament and center of the uterus were confirmed as well. There are lots of blood vessels including the cervix of uterus venous plexus at the lateral wall of uterus that increase the risk of ureter injury (-Fig. 2A).

At lower uterine segment, transverse incision is used safely in most instances. In the cases where uterine internal os opened and uterine effacement progressed, an appropriate myometrial incision line progresses upward. The height of the vesicouterine pouch may not be at appropriate line (-Fig. 2B). If the pelvis is too deep and seems to be hard to operate, transverse incision is performed without detachment of the peritoneum over the vesicouterine pouch.

The multifetal pregnancy has many cases that placenta is located at anterior wall and may result placenta previa. There are lots of thick blood vessels. Ligation of these is considered before the myometrium incision.

To extend a myometrium wound using a scissor is recommended after reach out at the membrane. We insert Pean's forceps between a membrane and a myometrium or pull up myometrium with a finger without rupture of the membrane. In addition, in case of rupture of the membrane, the scissor does not injure the fetuses. The incision line looks like $\mathbf{U}-$ shaped, near to $\mathbf{V}$-shaped, and is longer than singleton pregnancy. $\mathbf{U}$-shaped myometrium incision prevents injury to the vascular plexus of the uterine lateral wall. Then move the scissor forward to the beginning of the round ligament (-Fig. 3). Once the myometrium wound suturing is done, surprisingly it will not appear curved. Using a scissor is easy to 


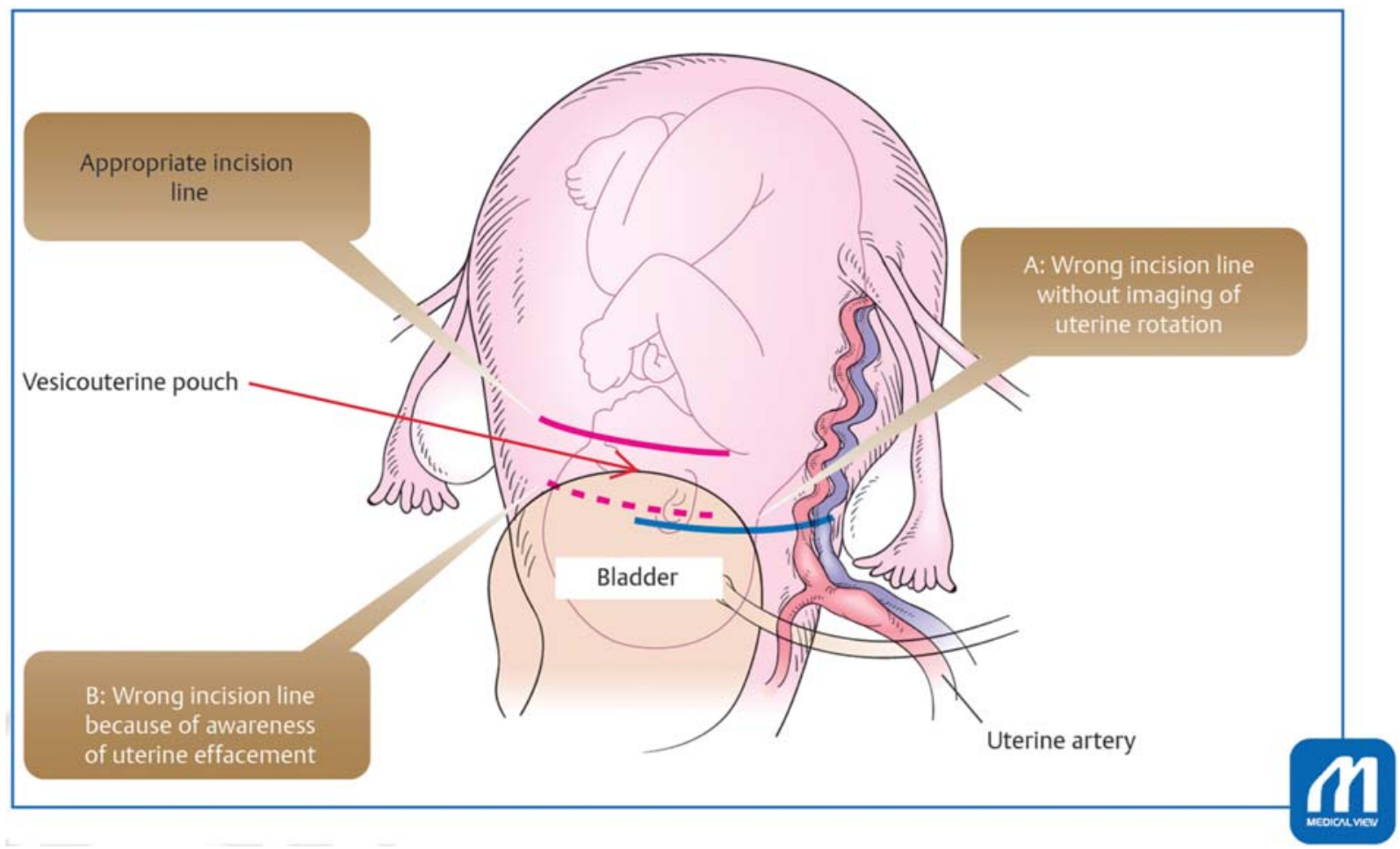

Fig. 2 Myometrium incision with imaging of uterine rotation. (Reproduced with permission from Tanigaki S, Iwashita M. Multifetal pregnancy. In: Hiramatsu Y, Konishi I, Sakuragi N, Takeda S, eds. Mastering the Essential Surgical Procedures OGS NOW, No.3. Cesarean Section (Japanese). Tokyo: Medical View; 2010: 56-63. Copyright @ Medical View).

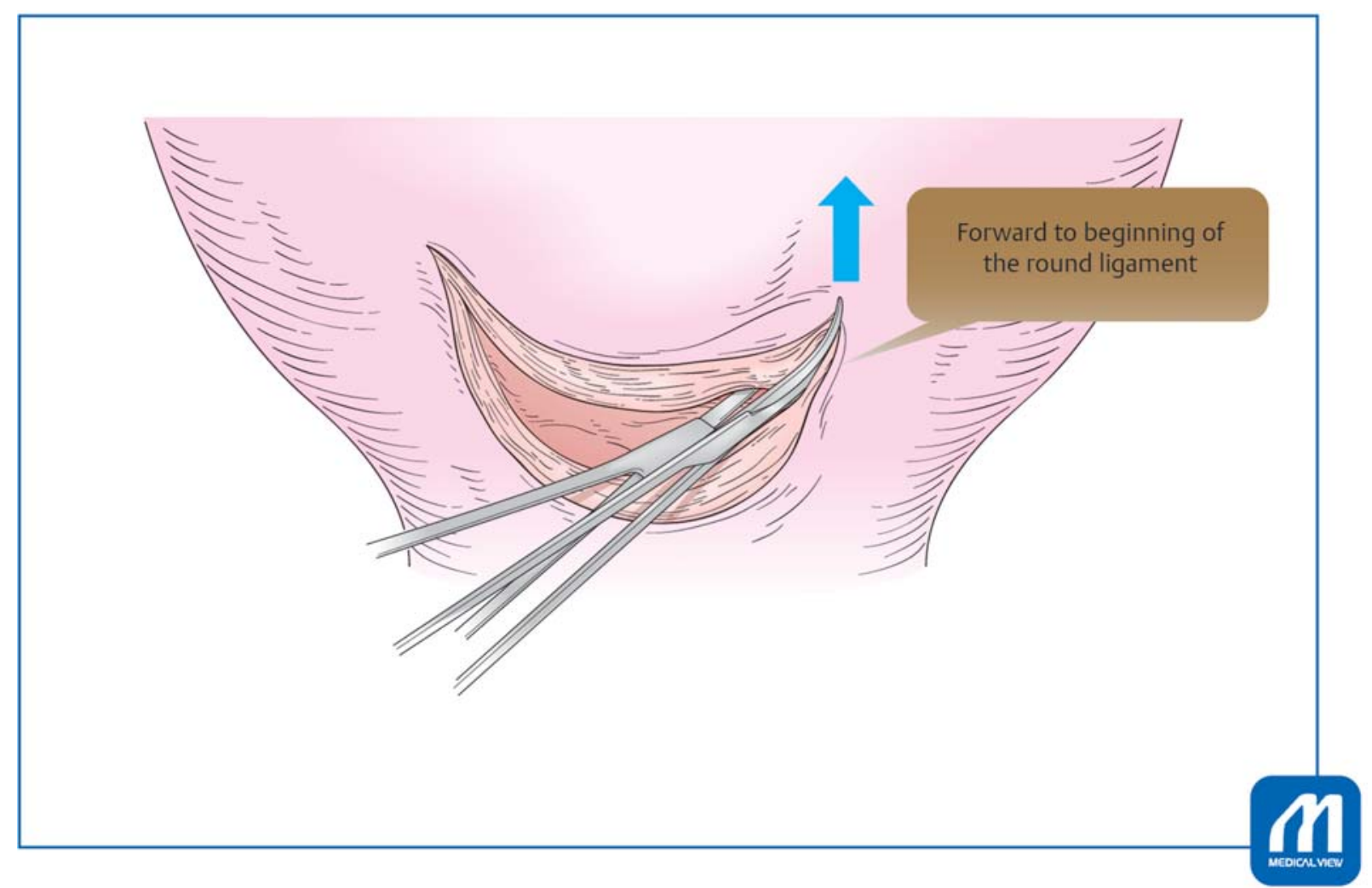

Fig. 3 U-shaped myometrium incision using a scissor and Pean's forceps forward to beginning of the round ligament. (Reproduced with permission from Tanigaki S, Iwashita M. Multifetal pregnancy. In: Hiramatsu Y, Konishi I, Sakuragi N, Takeda S, eds. Mastering the Essential Surgical Procedures OGS NOW, No.3. Cesarean Section (Japanese). Tokyo: Medical View; 2010: 56-63. Copyright @ Medical View). 
Table 2 Causes of PPH in multifetal pregnancy

\begin{tabular}{|l|l|}
\hline Uterine atony & $\begin{array}{l}\text { Hyperextension of uterus } \\
\text { Fatigue of myometrium: long usage of tocolytic agent including of magnesium } \\
\text { sulfate to management preeclampsia } \\
\text { Placenta located in the lower uterine segment: huge size of placenta }\end{array}$ \\
\hline Delay of myometrium suture & Delayed placenta delivery \\
\hline High risk of complications & High risk of disorder of blood coagulation: GT, PIATD, HELLP syndrome, acute fatty liver \\
\hline
\end{tabular}

Abbreviations: GT, gestational thrombocytopenia; HELLP, hemolysis, elevated liver enzymes, low platelet count; PIATD, pregnancy-induced antithrombin deficiency; PPH, postpartum hemorrhage.

(Reproduced with permission from Tanigaki S, Iwashita M. Multifetal pregnancy. In: Hiramatsu Y, Konishi I, Sakuragi N, Takeda S, eds. Mastering the Essential Surgical Procedures OGS NOW, No.3. Cesarean Section (Japanese). Tokyo: Medical View; 2010: 56-63. Copyright @ Medical View).

perform $\mathbf{J}$ - or $\mathbf{L}$-shaped incision and can also avoid myoma of the uterus and thick vessels. One has to learn to extend the wound with the help of finger properly. But in the case of emergency, using a scissor is not recommended without having usual training.
Delivery of the First Twin-Is the Fetus Really the First Twin?

The second twin makes the delivery of the first twin difficult. A longer myometrium incision is helpful. Do not rupture of the membrane immediately when

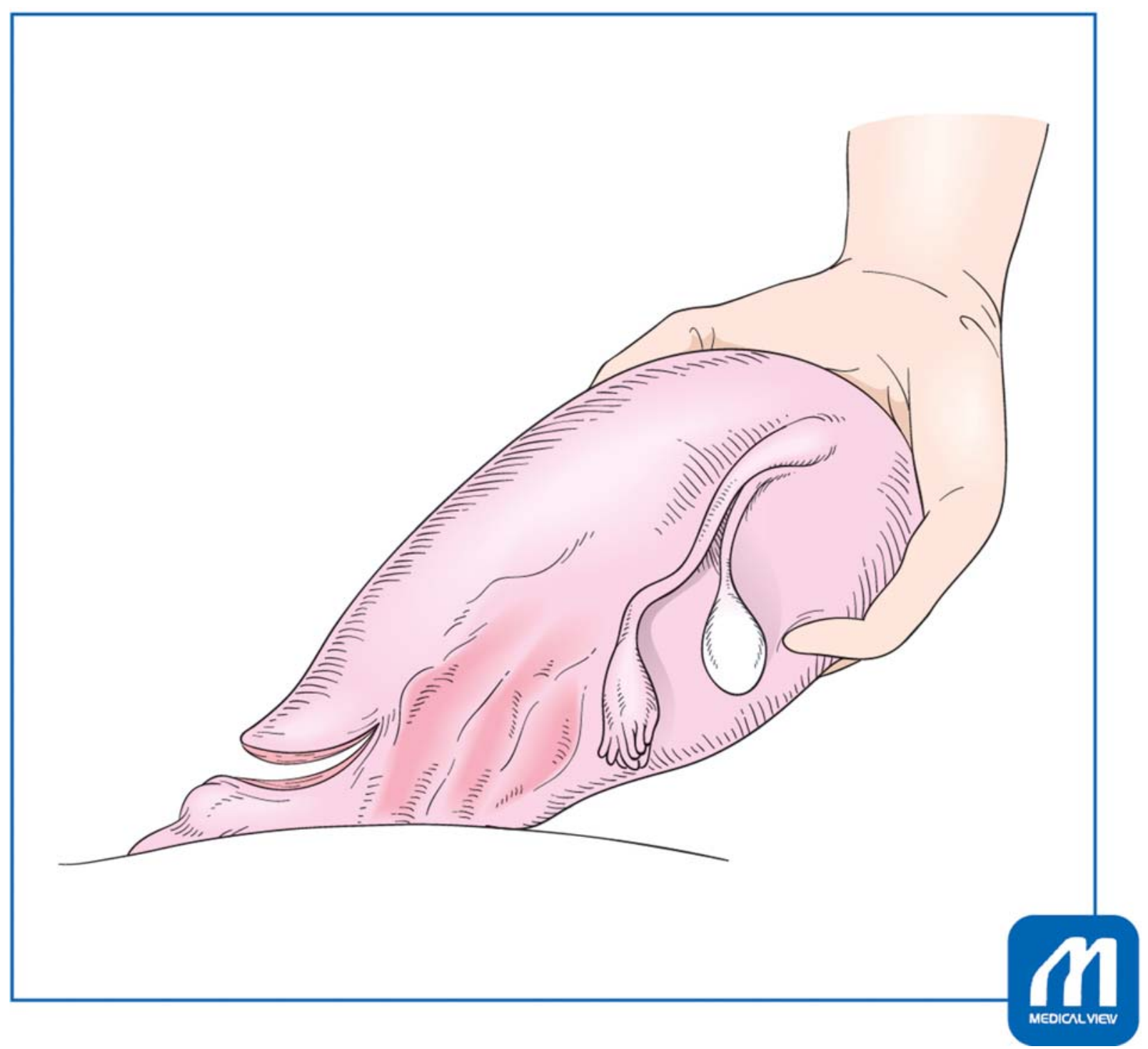

Fig. 4 Uterus is drawn the outside of the abdominal wall. (Reproduced with permission from Tanigaki S. Iwashita M. Multifetal pregnancy. In: Hiramatsu Y, Konishi I, Sakuragi N, Takeda S, eds. Mastering the Essential Surgical Procedures OGS NOW, No.3. Cesarean Section (Japanese). Tokyo: Medical View; 2010: 56-63. Copyright @ Medical View). 
membrane is visible. In the case of multifetal pregnancy, the membrane which is easy to rupture may be second twin's membrane. If the membrane of the second twin is ruptured before the first twin's delivery, operation of afterward is hard. Therefore, we have a pause, too. Check the both twins and, if delivery is possible, rupture of that fetus's membrane.

\section{Delivery of the Second Twin-Check the Presentation and Care of Entrapment}

After the first twin's delivery, keep the membrane of the second twin. There is always the risk that the second twin changes the presentation and the upper uterine segment will clamp down and entrap the second twin.

At first check the presentation of the second twin before rupture of that fetus's membrane. If the fetus is nonvertex, make intrauterine manipulation to footing presentation. It is difficult to hold the fetal head, whereas it is easy to hold the foot by palpating fetus's heel.

If contraction ring may develop the upper uterine segment, a vertical uterine incision is performed without hesitation. And uterine relaxation with 50 to $100 \mu \mathrm{g}$ intravenous nitroglycerine is considered. Because nitroglycerine takes 1 minute until an effect is given, it is prepared before starting the operation and early decision is necessary.

\section{Warnings}

\section{Prevention of Postpartum Hemorrhage}

Women with multifetal pregnancy are at increased risk of postpartum hemorrhage (PPH). As shown in - Table 2, causes of PPH in multifetal pregnancy.

Mainly PPH is caused by uterine atony. An oxytocin intravenous infusion or injection is performed immediately after delivery of the second twin. So oxytocin should be prepared before starting the $\mathrm{CD}$. Urgent manual removal of placentas causes remained placenta that can increase the amount of bleeding. After placenta delivery, uterine posterior wall is confirmed that it does not have the adhesion. Then uterus pulls up outside of the abdominal cavity ( - Fig. 4). The both uterine arteries are suppressed by the abdominal wall, and the bleeding from the myometrial wound is reduced. In addition, because bleeding does not accumulate in the wound, it is easy to get a field of vision and to myometrium suture. But it is important to train myometrium suture at intra-abdominal space. Because the uterus is huge or there are severe adhesions around the uterus, uterus cannot pull out the outside of the abdominal cavity. If hemostasis is insufficient, insertion of a balloon and gauze is recommended along with suture. Tourniquet technique to suppress uterine cervix and parametrium is a simple and an effective maneuver (-Fig. 5).

All bleeding may not be recognized in the operation field. Blood pressure and heart rate or shock index(SI; the heart rate/systolic blood pressure) may not show a state for the massive bleeding. We would like to cautious surgeons from losing the timing of the blood transfusion.

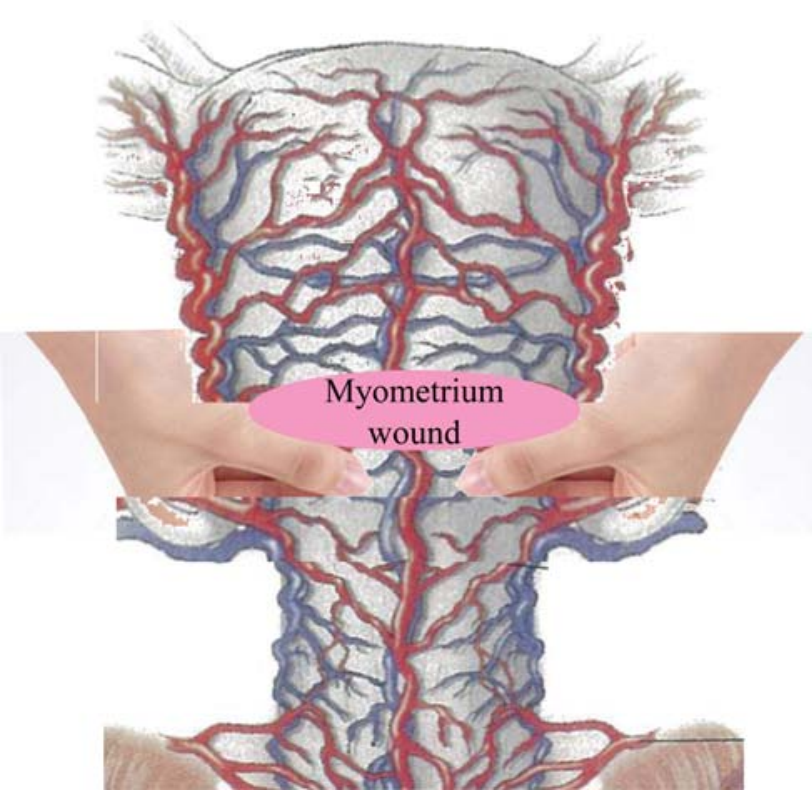

Fig. 5 Tourniquet technique. (Reproduced with permission from Dr. Shinji Tanigaki).

\section{Risk of Emergency Caesarean Section after Vaginal Delivery}

In the vaginal birth of the multifetal pregnancy, emergency $\mathrm{CD}$ is necessary for delivery of the second twin after VD of the first twin. The second twin does not fix in a pelvis after the prompt delivery of the first twin, then descending umbilical cord, malrotation, and abnormal presentation may occur. In addition, sustained bradycardia and abruption of placentae with sudden uterine contraction can be caused. As per literature, $9.45 \%$ of the second twin was performed emergency CD after VD of the first twin. ${ }^{9}$ The risk of the second twin performed by emergency CD after VD of the first twin, that is, vertex-vertex presentation, is approximately $7 \%$. But vertex-nonvertex presentation becomes higher with approximately $23 \%$ as for the risk. ${ }^{10}$ The prevalence (low 5minute Apgar's score, respirator management, and convulsions) rises for the second twin, if an emergency $C D$ was performed. ${ }^{9}$

Conflict of Interest

None declared.

\section{References}

1 Barrett J, Aztalos E, Willan A, et al. The twin birth study: a multicenter RCT of planned cesarean section (CS) and planned vaginal birth (VB) for twin pregnancies 320 to 386/7 weeks. Am J Obstet Gynecol 2013;208(1, Suppl):S4-S5

2 Hofmeyr GJ, Barrett JF, Crowther CA. Planned caesarean section for women with a twin pregnancy. Cochrane Database Syst Rev 2015;(12):CD006553

3 Hogle KL, Hutton EK, McBrien KA, Barrett JF, Hannah ME. Cesarean delivery for twins: a systematic review and meta-analysis. Am J Obstet Gynecol 2003;188(01):220-227

4 Barrett JF, Hannah ME, Hutton EK, et al; Twin Birth Study Collaborative Group. A randomized trial of planned cesarean or vaginal delivery for twin pregnancy. N Engl J Med 2013;369(14): 1295-1305 
5 Schmitz T, Carnavalet CdeC, Azria E, Lopez E, Cabrol D, Goffinet F. Neonatal outcomes of twin pregnancy according to the planned mode of delivery. Obstet Gynecol 2008;111(03): 695-703

6 Luke B. Reducing fetal deaths in multiple births: optimal birthweights and gestational ages for infants of twin and triplet births. Acta Genet Med Gemellol (Roma) 1996;45(03):333-348

7 Chasen ST, Madden A, Chervenak FA. Cesarean delivery of twins and neonatal respiratory disorders. Am J Obstet Gynecol 1999; 181(5, Pt 1):1052-1056
8 Minakami H, Kosuge S, Fujiwara H, Mori Y, Sato I. Risk of premature birth in multifetal pregnancy. Twin Res 2000;3(01):2-6

9 Wen SW, Fung Kee Fung K, Oppenheimer L, Demissie K, Yang Q, Walker M. Neonatal morbidity in second twin according to gestational age at birth and mode of delivery. Am J Obstet Gynecol 2004;191(03):773-777

10 Wen SW, Fung KF, Oppenheimer L, Demissie K, Yang Q, Walker M. Occurrence and predictors of cesarean delivery for the second twin after vaginal delivery of the first twin. Obstet Gynecol 2004; 103(03):413-419 